\title{
Pheochromocytoma due to a novel SDHD variant presenting as unilateral visual loss
}

\author{
Clare Miller1', Agnieszka Pazderska', John Reynolds², Patricia Gou³, Barbara Dunne ${ }^{3}$, \\ Kealan McElhinney ${ }^{4}$ and Lisa Owens ${ }^{1}$
}

1Department of Endocrinology, 2Department of Surgery, 3Department of Histopathology, St. James's Hospital, Dublin, Ireland, and ${ }^{4}$ Department of Ophthalmopathy, Royal Victoria Eye and Ear Hospital, Dublin, Ireland
Correspondence should be addressed to C Miller

Email

clarem4@gmail.com

\section{Summary}

A 53-year-old female presented to a tertiary ophthalmology referral centre complaining of unilateral painless loss of vision. Subsequent assessment revealed malignant hypertension causing right-sided cystoid macular oedema. During the course of secondary hypertension workup, she was diagnosed with a $7.8 \mathrm{~cm}$ phaeochromocytoma which was resected. Testing for a panel of all predisposing phaeochromocytoma-causing variants using next-generation sequencing resulted in the diagnosis of a novel SDHD variant.

\section{Learning points}

- Screening for secondary causes of hypertension is indicated when there is evidence of hypertension-mediated end-organ damage (1).

- Testing for a predisposing variant should be considered in all patients with phaeochromocytoma or paraganglioma due to the high heritability rate and prevalence of somatic variants $(2,3,4)$.

- Novel variants are commonly uncovered in the Succinate Dehydrogenase (SDH) subunit; proving pathogenicity is a complex, time-consuming process and one challenge of next-generation sequencing (3).

- SDHB immunohistochemistry as a tool for demonstrating pathogenicity is associated with reduced sensitivity when assessing SDHD variants $(5,6)$.

\section{Background}

Phaeochromocytoma (PC) and paraganglionoma (PGL) are rare neuroendocrine tumours arising in chromaffin cells of the adrenal medulla or extra-adrenal chromaffin cells, respectively (2). The majority of phaeochromocytoma and paraganglioma (PPGL) are diagnosed due to incidental uncovering of an adrenal lesion in the course of imaging for alternate indications or during surveillance in patients with a known genetic predisposition (7). Symptomatic presentation typically comprises paroxysmal palpitations, anxiety, sweating and headaches, though the prevalence of PPGL is low in those tested for signs and symptoms only (7). Patients rarely present with a life-threatening catecholamine crisis (2).
PPGL demonstrates the highest heritability rate of all tumours. Over the last decade, more than 15 predisposing variants have been discovered (3). If both sporadic and germline variants are considered, $50 \%$ of PPGL possess an underlying predisposing variant (2, $3,4)$. However in most centres genetic testing remains restricted to patients with the following risk factors as per the NHS National Genomic Test Directory - diagnosis of PPGL at age $<60$ years, extra-adrenal disease, PPGL with loss of staining for Succinate dehydrogenase B (SDHB) on immunohistochemistry (IHC), bilateral PC, PC and renal cell carcinoma (RCC), PPGL and $>1$ relative with PPGL/RCC (3). 

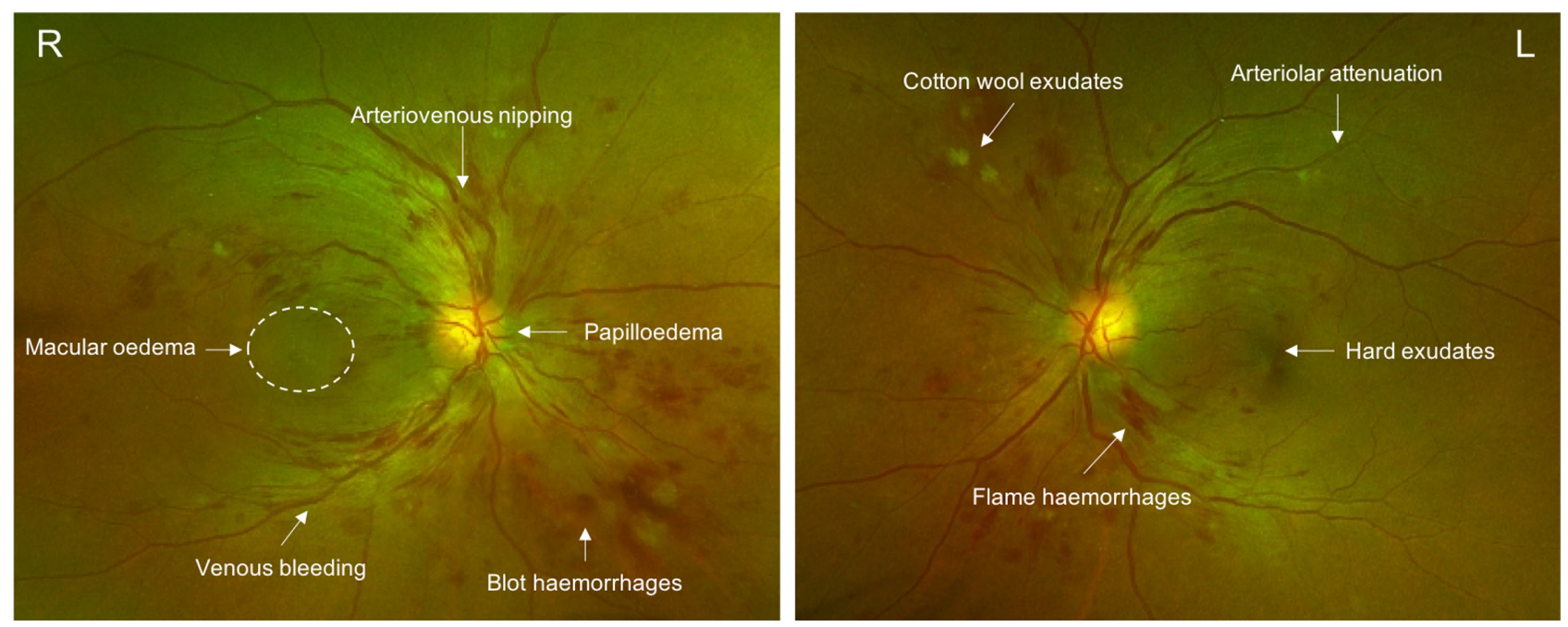

Figure 1

Colour fundus photo of both eyes demonstrating grade 4 hypertensive retinopathy (Keith Wagener Barker Classification).

\section{Case presentation}

A 53-year-old Nigerian female was referred for investigation and management of bilateral hypertensive retinopathy by ophthalmology. She described 2 weeks of painless right-sided 'foggy' reduction in her visual acuity. On examination, she could finger count only on the right and had normal visual acuity at 6/6 on the left. Examination by ophthalmology revealed bilateral hypertensive retinopathy, with cystoid macular oedema in the right eye only (Fig. 1). Her initial blood pressure was 218/111 mmHg in the Emergency Department, antihypertensive therapy was initiated immediately. Her medical history was significant for hepatitis B and she did not take any regular medications. There was no relevant family history. A detailed review of symptoms reveals intermittent episodes of sweating attributed to a perimenopausal status. Given her presentation and severe hypertension, secondary hypertension workup was initiated.

\section{Investigation}

An overnight dexamethasone suppression test and aldosterone renin ratio prior to the introduction of antihypertensive medications ruled out cortisol and aldosterone excess (Table 1). Magnetic resonance angiogram of the renal arteries performed with the intention of ruling out renal artery stenosis reported leftsided adrenal mass. Further clarification with a CT thorax abdomen and pelvis confirmed a $5.4 \times 6.2 \times 7.8 \mathrm{~cm}$ left adrenal mass with a central necrotic/cystic like appearance and a mildly enhancing wall, features consistent with a pheochromocytoma with no evidence of extra-adrenal metastasis (Fig. 2). Plasma metanephrines showed grossly elevated metanephrine and normetanephrine concentrations consistent with the suspected diagnosis of PC (Table 2). A pre-operative echocardiogram demonstrated moderate concentric left ventricular hypertrophy (LVH) and mild diastolic dysfunction.

\section{Treatment}

She proceeded to left adrenalectomy 11 days postadmission following alpha-blockade and salt loading. She was monitored in the intensive care unit for $48 \mathrm{~h}$ postoperatively. Repeat plasma metanephrines 6 weeks postresection confirmed complete resolution of catecholamine excess (Table 3).

\section{Outcome and follow-up}

Histopathological examination of the resected tumour confirmed the presence of pheochromocytoma (Fig. 3) with positive staining for $\mathrm{S} 100$ and chromogranin (Fig. 4). The Pheochromocytoma of the Adrenal gland Scaled Score

Table 1 Secondary hypertension workup.

\begin{tabular}{lll}
\hline Secondary hypertension workup & \\
\hline $\begin{array}{l}\text { Cortisol (post-1 mg } \\
\text { dexamethasone) }\end{array}$ & $45 \mathrm{nmol} / \mathrm{L}$ & $<50 \mathrm{nmol} / \mathrm{L}$ \\
Aldosterone & $316 \mathrm{pmol} / \mathrm{L}$ & $\begin{array}{c}\text { Supine 102-859 } \\
\mathrm{pmol} / \mathrm{L}\end{array}$ \\
Direct renin & $21 \mathrm{mIU} / \mathrm{L}$ & $\begin{array}{c}\text { Supine 4.2-59.7 } \\
\mathrm{mIU} / \mathrm{L}\end{array}$ \\
\hline
\end{tabular}




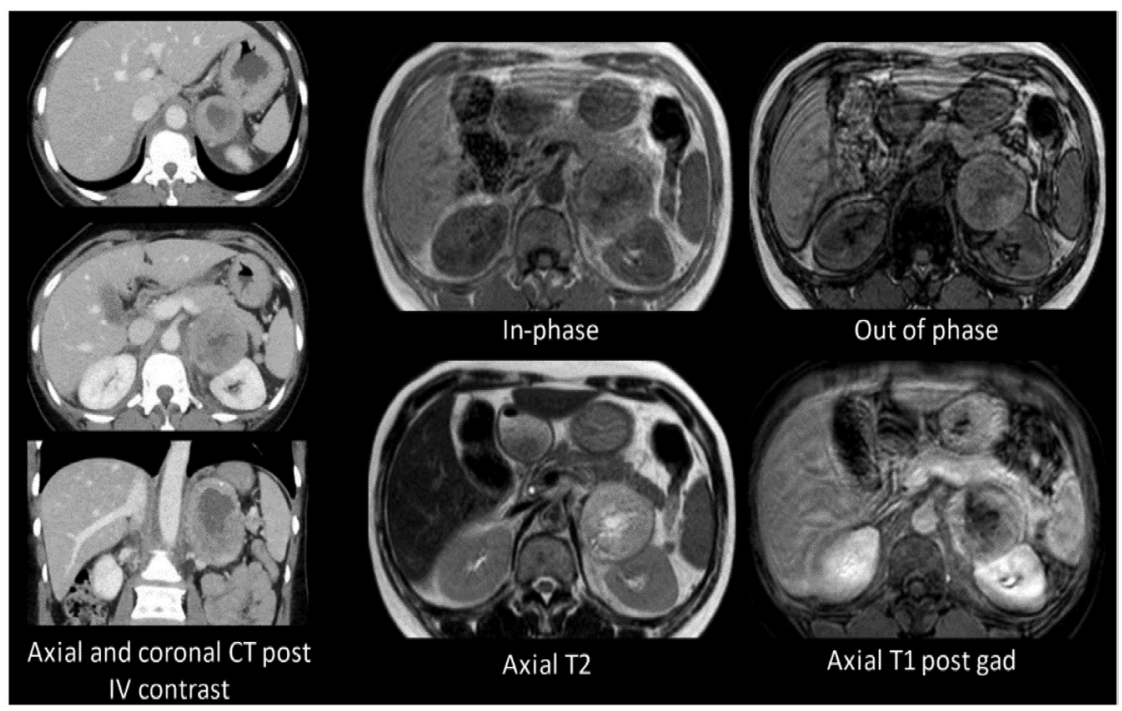

\section{Figure 2}

CT of thorax abdomen and pelvis.
(PASS) was 4. SDHB expression was retained with positive granular and cytoplasmic staining. Genetic analysis was carried out via next-generation sequencing (NGS) for a panel of 11 predisposing variants (FH, MAX, MEN1, RET, SDHA, SDHB, SDHC, SDHD, SDHAF2, TEMEM127 and VHL). This confirmed mosaicism for a novel variant. Deletion of exon 1 of the SDHD gene located at chromosome 11 was predicted to result in loss of $57 \%$ of the SDHD protein (Fig. 5). The level of mosaicism for the SDHD exon 1 deletion within her leukocyte DNA was estimated at least 30\% consistent with a post-zygotic variant. Her children were referred to clinical genetics for counselling, and for her male descendants testing for the specific variant identified. Lifelong surveillance will include annual clinical assessment and plasma metanephrines. An MRI of neck, thorax, abdomen and pelvis is pending 1 year post-resection of PC and if normal will be performed on an ongoing 2-yearly basis. She now requires ramipril only for essential hypertension and her visual acuity has normalised.

\section{Discussion}

This case highlights the importance of assessing for secondary causes of hypertension and the application of genetics. Current guidelines suggest consideration of workup in the following instances: patients $<40$ years, acute

Table 2 Pre-operative plasma metanephrines.

\begin{tabular}{ll}
\hline Plasma metanephrines & Reference range \\
\cline { 1 - 2 } Metanephrine & $>7000 \mathrm{pmol} / \mathrm{L}(61-377)$ \\
Normetanephrine & $>10000 \mathrm{pmol} / \mathrm{L}(182-867)$ \\
3-Methoxytyramine & $539 \mathrm{pmol} / \mathrm{L}(<185)$ \\
\hline
\end{tabular}

worsening of previously stable hypertension, drug-resistant hypertension, hypertension-mediated end-organ damage, clinical/biochemical features suggestive of an endocrine cause, hypertensive emergency and if suggestive clinical features are present investigation of obstructive sleep apnoea (1). The prevalence of PC is significantly higher in patients who present with hypertension than the general population, however, it remains a rare neoplasm (8). Screening for PPGL using plasma or urinary metanephrines is advised in all instances of secondary hypertension workup $(1,2)$.

In this case, end-organ damage of long-standing hypertension (hypertensive retinopathy and LVH) were the only indicators of PC and the classic triad of paroxysmal headaches, sweating and palpitations were confirmed by the patient following retrospective review of history. It is now recognised that PCs are most commonly diagnosed following uncovering of an adrenal incidentaloma or detection of asymptomatic cases in patients screened due to first-degree relatives with predisposing PPGL-causing variants. The retrospective recognition of symptoms, in this case, is commonplace (7). PC presenting as visual loss has been described previously and highlights the importance of multi-disciplinary engagement in management of these rare tumours (9).

The majority of PPGL-causing variants occur in the SDHx gene (2). The SDHx enzyme catalyses the oxidation

Table 3 Post-operative plasma metanephrines.

Plasma metanephrines

Metanephrine

Normetanephrine

3-Methoxytyramine
Reference range

$140 \mathrm{pmol} / \mathrm{L}(61-377)$

$678 \mathrm{pmol} / \mathrm{L}(182-867)$

$<65 \mathrm{pmol} / \mathrm{L}(<185)$ 

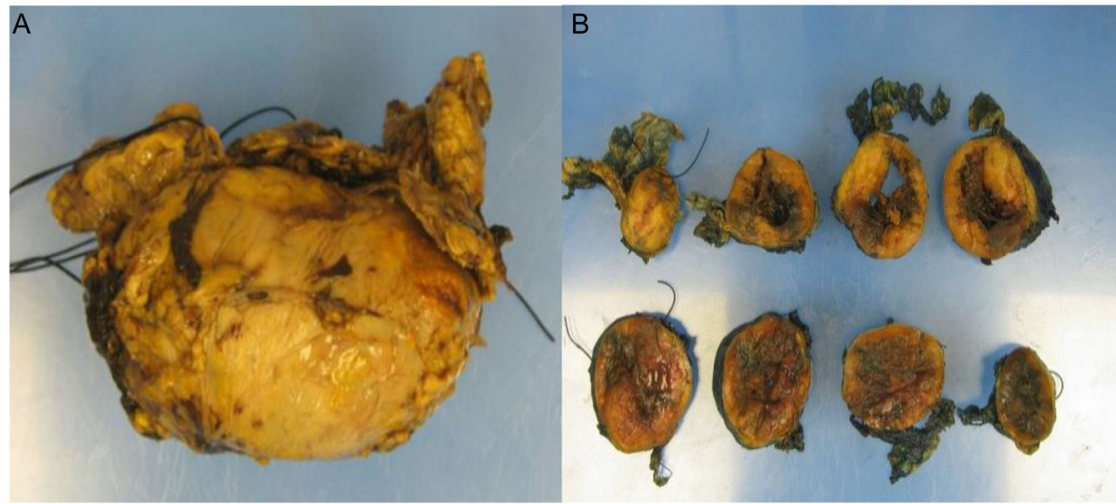

\section{Figure 3}

Gross resected tumour tissue (A) left adrenal gland $110 \mathrm{~g}$ (B) Cross-section of adrenal gland reveals a well-defined yellow lesion with a haemorrhagic centre. There is minimal normal adrenal tissue seen.

of succinate to fumarate in the citric acid cycle and the transfer of electrons to the ubiquinone pool in the electron transport chain. A variant affecting the function of any SDH subunit (A-D) culminates in loss of SDH enzymatic activity and accumulation of succinate which is the main driver of SDHx tumourigenesis (3). In this case, a novel SDH subunit variant was uncovered.

Assigning pathogenicity to a novel variant is a complex process that must be rigorously applied as it will impact on surveillance duration, choice of imaging modalities and in the future probably targeted gene therapies for both the patient and their family $(3,4)$.

The process of determining the role of a novel variant in pathogenesis of a disease requires multiple lines of supporting evidence outlined by the American College of Medical Genetics and Genomics (ACMG) as demonstrated in Fig. 6 (10). This process has garnered attention among endocrinologists over the last number of years as NGS is now best practice when screening for variants causing
PPGL. NGS is more efficient from a time and cost perspective than the now outdated Sanger sequencing but results in a larger data output and a greater workload detangling the pathogenicity of novel variants and variants of unknown significance $(2,3,4)$.

First, population databases can be referenced to determine the frequency of a suspected pathogenic variant in control populations. The SDHD variant isolated in this case has never been previously identified in the gnomAS or DGV databases which contain variants from healthy population cohorts. Population data is considered moderate evidence of pathogenicity if a variant is absent in general population $(4,10)$.

Computational tools aid in assessing the predicted impact of a variant on protein structure and function. In this case, the probability of a non-functional SDHD complex was based on the location of the novel variant in the context of the protein sequence. Dosage analysis of the SDHD gene by Drop Digital PCR confirmed deletion of
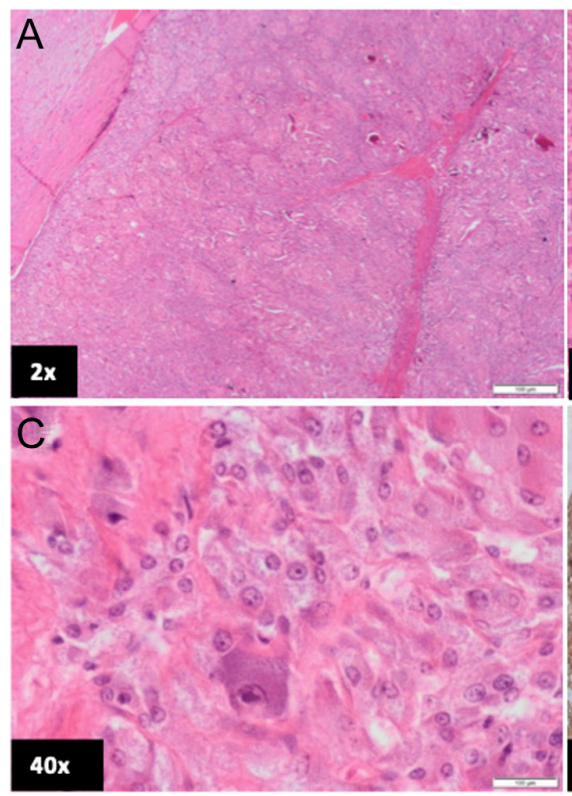
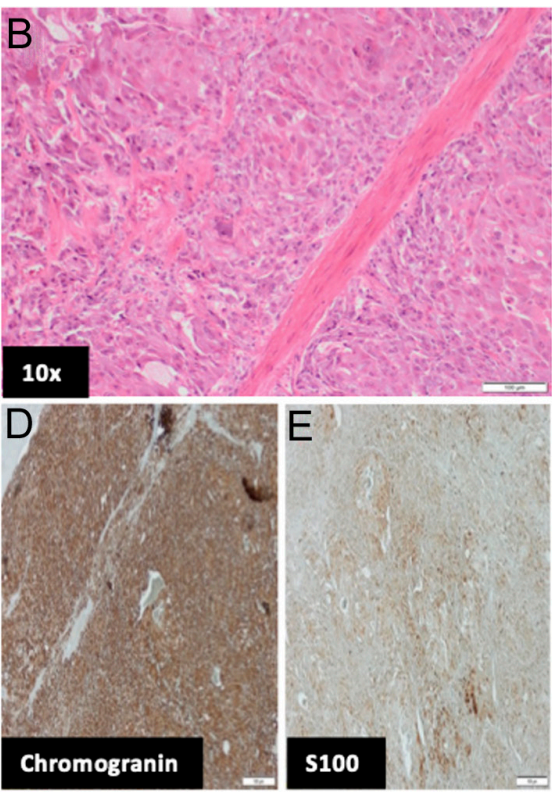

\section{Figure 4}

Histopathologic findings of the resected tumour consistent with phaeochormocytoma. Histology shows nests of epithelioid cells with prominent nucleoli and eosinophilic, some foamy cytoplasm with interspersed small blood vessels at increasing magnification $(A, B, C)$ Necrosis is not present and there is no lymphovascular invasion. There is positive chromogranin staining (D) and S100 staining for sustentacular cells (E). 


\begin{tabular}{|l|l|l|l|l|l|}
\hline Gene & Zygosity & Inheritance & HGVS description & Location: GRCh37 (hg19) & $\begin{array}{l}\text { Classificatio } \\
n\end{array}$ \\
\hline SDHD & Mosaic & Not known & $\begin{array}{l}\text { NM_003002.2:c.(-383_-88) } \\
\text { (52+1_53-1)del p.? } \\
\text { Deletion of SDHD exon 1 }\end{array}$ & $\begin{array}{l}\text { Chr11:g.(111957249_111957544)_ } \\
\left(111957684 \_111958580\right) \text { del }\end{array}$ & $\begin{array}{l}\text { Likely } \\
\text { Pathogenic }\end{array}$ \\
\hline
\end{tabular}

Figure 5

Variant details.

SDHD exon 1. The next in-frame initiation codon is located in exon 3 and would result in the loss of $\sim 57 \%$ of the SDHD protein. This in vivo demonstration of an impact on protein function as per the ACMG is considered strong evidence of pathogenicity (10).

SDHB Immunochemistry (IHC) is a well-validated tool for assessing pathogenicity among SDHx subunit variants (11). A pathogenic variant affecting any SDHx subunit results in destabilisation of the anchor SDHB protein and a characteristic absent SDHB mitochondrial granular staining pattern or alternatively a weak diffuse staining pattern (11). Absent SDHB staining is supportive of impaired gene expression and is therefore a 'strong' criterion supportive of pathogenicity as per the ACMG (10, 11). In this case, there was preserved SDHB IHC granular and cytoplasmic staining of tumour tissue prohibiting classification of this variant as pathogenic as opposed to likely pathogenic.

Interpretation of SDHB staining via IHC is less sensitive in the case of SDHD mutated tumours relative to SDHB due to the weak diffuse signal pattern sometimes described (5, $6,11)$. Reporting should be carried out in expert centres by pathologists with significant experience reporting SDHB IHC.

In conclusion based on ACMG criteria, the novel variant identified in this case is likely pathogenic (Fig. 7, point (ii)) (10). SDHD gene is located on chromosome 11, comprises four exons and the protein anchors the SDH complex to the inner mitochondrial membrane. SDHD variants present most commonly as head and neck PGL (HNPGL) $(3,12)$. It is hypothesised that the chronic stimulation of hypoxic signalling factors associated with SDHx variants is responsible for the greater association with HNPGL, as populations living at higher altitude have a greater prevalence of carotid body tumours (13). In this case, a novel variant predicted to cause deletion of SDHD exon 1 presented as a PC. Previous studies have highlighted the association between variant and clinical presentation and particularly relevant to this case is the increased incidence of PC relative to HNPGL in patients with an SDHD truncating vs missense variant (14). Perhaps if this is the case there is a novel mechanism of tumourigenesis present in this patient presenting with PC and a novel truncating SDHD variant. SDHx mutations demonstrate autosomal dominant transmission however uniquely SDHD, SDHAF2 and MAX variants

\begin{tabular}{|c|c|}
\hline Pathogenic & $\begin{array}{l}\text { (i) } 1 \text { Very strong (PVS1) AND } \\
\text { (a) } \geq 1 \text { Strong (PS1-PS4) OR } \\
\text { (b) } \geq 2 \text { Moderate (PM1-PM6) OR } \\
\text { (c) } 1 \text { Moderate (PM1-PM6) and } 1 \text { supporting } \\
\text { (PP1-PP5) OR } \\
\text { (d) } \geq 2 \text { Supporting (PP1-PP5) } \\
\text { (ii) } \geq 2 \text { Strong (PS1-PS4) OR } \\
\text { (iii) } 1 \text { Strong (PS1-PS4) AND } \\
\text { (a) } \geq 3 \text { Moderate (PM1-PM6) OR } \\
\text { (b) } 2 \text { Moderate (PM1-PM6) AND } \geq 2 \\
\text { Supporting (PP1-PP5) OR } \\
\text { (c)1 Moderate (PM1-PM6) AND } \geq 4 \\
\text { supporting (PP1-PP5) }\end{array}$ \\
\hline Likely pathogenic & $\begin{array}{l}\text { (i) } 1 \text { Very strong (PVS1) AND } 1 \text { moderate (PM1- } \\
\text { PM6) OR } \\
\text { (ii) } 1 \text { Strong (PS1-PS4) AND 1-2 moderate } \\
\text { (PM1-PM6) OR } \\
\text { (iii) } 1 \text { Strong (PS1-PS4) AND } \geq 2 \text { supporting } \\
\text { (PP1-PP5) OR } \\
\text { (iv) } \geq 3 \text { Moderate (PM1-PM6) OR } \\
\text { (v) } 2 \text { Moderate (PM1-PM6) AND } 2 \text { supporting } \\
\text { (PP1-PP5) OR } \\
\text { (vi) } 1 \text { Moderate (PM1-PM6) AND } \geq 4 \text { supporting } \\
\text { (PP1-PP5) }\end{array}$ \\
\hline Benign & $\begin{array}{l}\text { (i) } 1 \text { Stand-alone (BA1) OR } \\
\text { (ii) } \geq 2 \text { Strong (BS1-BS4) }\end{array}$ \\
\hline Likely benign & $\begin{array}{l}\text { (i) } 1 \text { Strong (BS1-BS4) and } 1 \text { supporting (BP1- } \\
\text { BP7) OR } \\
\text { (ii) } \geq 2 \text { Supporting (BP1-BP7) }\end{array}$ \\
\hline $\begin{array}{l}\text { Uncertain } \\
\text { significance }\end{array}$ & $\begin{array}{l}\text { (i) Other criteria shown above are not met } O R \\
\text { (ii) the criteria for benign and pathogenic are } \\
\text { contradictory }\end{array}$ \\
\hline
\end{tabular}

Figure 6

Standards and guidelines for the interpretation of sequence variants: American College of Medical Genetics and Genomics and the Association for Molecular Pathology (9). 


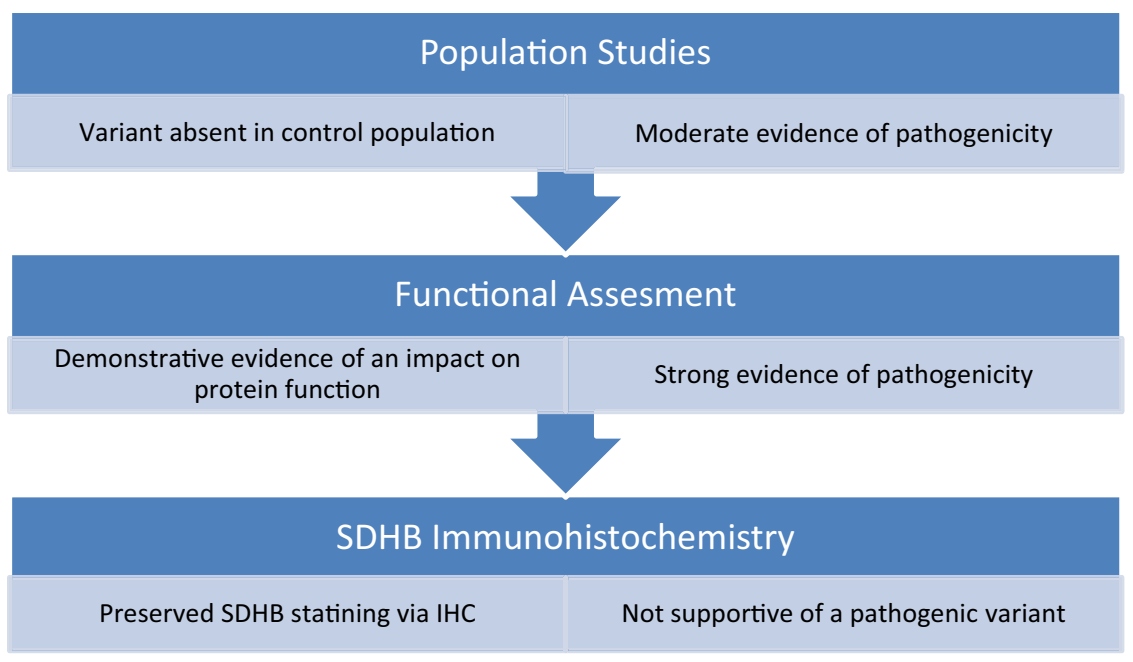

\section{Figure 7}

Biomarkers employed to demonstrate evidence of pathogenicity. possess a parent of origin effect whereby expression of disease is dependent on paternal transmission. Tumour development in SDHD mutated cases is hypothesized to require a three-hit model including either loss or mutation of the dominant SDHD allele and loss of the maternally active tumour suppressor gene. The only described case of tumour presentation in a maternally inherited SDHD mutation occurred due to a coexistent loss of the wildtype paternal SDHD allele and loss of heterozygosity affecting the maternal chromosome (15). As a result, at present, maternally inherited SDHD alleles are not sought out with genetic testing and if detected patients are not placed in surveillance programme (3).

Genetic screening in this case enabled focused precise genetic counselling for her male descendants only. For female offspring advice was given to seek endocrinology input for future generations only. Her parents and firstdegree relatives do not require assessment as this is a somatic variant (4).

In summary, this is a case of unilateral PC presenting as unilateral visual loss due to a novel likely pathogenic SDHD variant. The only clinical feature predictive of an underlying genetic mutation was age, suggesting that the possibility of a predisposing variant should be considered in all patients with PPGL. This case also highlights the complexity associated with assigning clinical significance to the large data output resulting from NGS and the importance of ensuring a thorough secondary hypertension workup is carried out when indicated to avoid missing life-threatening diagnosis.

\section{Declaration of interest}

The authors declare that there is no conflict of interest that could be perceived as prejudicing the impartiality of the research reported.

\section{Funding}

This work did not receive any specific grant from any funding agency in the public, commercial or not-for-profit sector.

\section{Patient consent}

Written informed consent has been obtained from the patient for publication of the case report and accompanying images.

\section{Author contribution statement}

Clare Miller prepared the manuscript and performed a literature review. Kealan McElhinney provided input from an ophthalmology perspective. Professor John Reynolds performed adrenalectomy and critically reviewed manuscript. Agnieszka Pazderska critically reviewed manuscript. Dr Lisa Owens was treating endocrinologist and critically reviewed manuscript.

\section{References}

1 Williams B, Mancia G, Spiering W, Rosei EA, Azizi M, Burnier M, Clement DL, Coca A, de Simone G, Dominiczak A, et al. 2018 ESC/ ESH guidelines for the management of arterial hypertension. The task force for the management of arterial hypertension of the European Society of Cardiology (ESC) and the European Society of Hypertension (ESH). Giornale Italiano di Cardiologia 201819 (Supplement 1) 3S-73S. (https://doi.org/10.1714/3026.30245)

2 Lenders JW, Duh QY, Eisenhofer G, Gimenez-Roqueplo AP, Grebe SK, Murad MH, Naruse M, Pacak K, Young WF \& Endocrine Society. Pheochromocytoma and paraganglioma: an Endocrine Society clinical practice guideline. Journal of Clinical Endocrinology and Metabolism 201499 1915-1942. (https://doi.org/10.1210/jc.2014-1498)

3 Casey R, Neumann HPH \& Maher ER. Genetic stratification of inherited and sporadic phaeochromocytoma and paraganglioma: implications for precision medicine. Human Molecular Genetics 2020 29 R128-R137. (https://doi.org/10.1093/hmg/ddaa201)

4 NGS in PPGL (NGSnPPGL) Study Group, Toledo RA, Burnichon N, Cascon A, Benn DE, Bayley JP, Welander J, Tops CM, Firth H, Dwight $\mathrm{T}$, et al. Consensus statement on next-generation-sequencingbased diagnostic testing of hereditary phaeochromocytomas and paragangliomas. Nature Reviews: Endocrinology 201713 233-247. (https://doi.org/10.1038/nrendo.2016.185) 
5 Santi R, Rapizzi E, Canu L, Ercolino T, Baroni G, Fucci R, Costa G, Mannelli M \& Nesi G. Potential pitfalls of SDH immunohistochemical detection in paragangliomas and phaeochromocytomas harbouring germline SDHx gene mutation. Anticancer Research 201737 805-812. (https://doi.org/10.21873/anticanres.11381)

6 Castelblanco E, Santacana M, Valls J, de Cubas A, Cascón A, Robledo M $\&$ Matias-Guiu X. Usefulness of negative and weak-diffuse pattern of SDHB immunostaining in assessment of SDH mutations in paragangliomas and pheochromocytomas. Endocrine Pathology 2013 24 199-205. (https://doi.org/10.1007/s12022-013-9269-4)

7 Falhammar H, Kjellman M \& Calissendorff J. Initial clinical presentation and spectrum of pheochromocytoma: a study of 94 cases from a single center. Endocrine Connections 20187 186-192. (https:// doi.org/10.1530/EC-17-0321)

8 Young WF, Calhoun DA, Lenders JWM, Stowasser M \& Textor SC. Screening for endocrine hypertension: an endocrine society scientific statement. Endocrine Reviews 201738 103-122.(https://doi. org/10.1210/er.2017-00054)

9 Banerjee A, Nayak B, Verma G \& Parija S. Resolution of grade IV hypertensive retinopathy in an adult with pheochromocytoma: post-tumor resection. BMJ Case Reports 202013 e231245. (https://doi. org/10.1136/bcr-2019-231245)

10 Richards S, Aziz N, Bale S, Bick D, Das S, Gastier-Foster J, Grody WW, Hegde M, Lyon E, Spector E, et al. Standards and guidelines for the interpretation of sequence variants: a joint consensus recommendation of the American College of Medical Genetics and Genomics and the Association for Molecular Pathology. Genetics in Medicine 201517 405-424. (https://doi.org/10.1038/gim.2015.30)
11 Gill AJ, Benn DE, Chou A, Clarkson A, Muljono A, Meyer-Rochow GY, Richardson AL, Sidhu SB, Robinson BG \& Clifton-Bligh RJ. Immunohistochemistry for SDHB triages genetic testing of SDHB, SDHC, and SDHD in paraganglioma-pheochromocytoma syndromes. Human Pathology 201041 805-814. (https://doi.org/10.1016/j. humpath.2009.12.005)

12 Andrews KA, Ascher DB, Pires DEV, Barnes DR, Vialard L, Casey RT, Bradshaw N, Adlard J, Aylwin S, Brennan P, et al. Tumour risks and genotype-phenotype correlations associated with germline variants in succinate dehydrogenase subunit genes SDHB, SDHC and SDHD. Journal of Medical Genetics 201855 384-394. (https://doi.org/10.1136/ jmedgenet-2017-105127)

13 Astrom K, Cohen JE, Willett-Brozick JE, Aston CE \& Baysal BE. Altitude is a phenotypic modifier in hereditary paraganglioma type 1 : evidence for an oxygen-sensing defect. Human Genetics 2003113 228-237. (https://doi.org/10.1007/s00439-003-0969-6)

14 Bayley JP, Bausch B, Rijken JA, van Hulsteijn LT, Jansen JC, Ascher D, Pires DEV, Hes FJ, Hensen EF, Corssmit EPM, et al. Variant type is associated with disease characteristics in SDHB, SDHC AND SDHD-linked phaeochromocytomaparaganglioma. Journal of Medical Genetics 202057 96-103. (https://doi.org/10.1136/ jmedgenet-2019-106214)

15 Yeap PM, Tobias ES, Mavraki E, Fletcher A, Bradshaw N, Freel EM, Cooke A, Murday VA, Davidson HR, Perry CG, et al. Molecular analysis of pheochromocytoma after maternal transmission of SDHD mutation elucidates mechanism of parent-oforigin effect. Journal of Clinical Endocrinology and Metabolism 201196 E2009-E2013. (https:// doi.org/10.1210/jc.2011-1244)

Received in final form 11 October 2021

Accepted 2 November 2021 